\title{
ARTICLE
}

Behavior, Psychology and Sociology

\section{Inhibition of food craving is a metabolically active process in the brain in obese men}

\author{
Gene-Jack Wang ${ }^{1} \cdot$ Ehsan Shokri Kojori $\mathbb{1}^{1} \cdot$ Kai Yuan $\mathbb{D}^{1,2} \cdot$ Corinde E. Wiers $^{1} \cdot$ Peter Manza' \\ Christopher T. Wong $\mathbb{D}^{1} \cdot$ Joanna S. Fowler ${ }^{3} \cdot$ Nora D. Volkow $\mathbb{1}^{1,4}$
}

Received: 5 March 2019 / Revised: 29 August 2019 / Accepted: 18 September 2019 / Published online: 18 November 2019

(c) The Author(s) 2019. This article is published with open access

\begin{abstract}
Objective Obesity is associated with impaired inhibitory control over food intake. We hypothesized that the neural circuitry underlying inhibition of food craving would be impaired in obesity. Here we assessed whether obese men show altered brain responses during attempted cognitive inhibition of craving when exposed to food cues.

Methods Sixteen obese men $(32 \pm 8.7$ years old, $\mathrm{BMI}=38.6 \pm 7.2)$ were compared with 11 age-matched non-obese men (BMI 24.2 \pm 2.5 ) using PET and FDG. Brain glucose metabolism was evaluated in a food deprived state: no food stimulation, food stimulation with no inhibition (NI), and food stimulation with attempted inhibition (AI), each on a separate day. Individualized favorite food items were presented prior to and after FDG injection for 40 min. For AI, participants were asked to attempt to inhibit their desire for the food presented. Self-reports for hunger and food desire were recorded.

Results Food stimulation compared with no stimulation increased glucose metabolism in inferior and superior frontal gyrus, default mode network and cerebellum, in both groups. For both groups, AI compared with NI-suppressed metabolism in right subgenual anterior cingulate, orbitofrontal areas, bilateral insula, and temporal gyri. There was a stimulation-by-group interaction effect in obese (but not in non-obese) men showing increased metabolism in pregenual anterior cingulate cortex (pgACC) and caudate during AI relative to NI. Changes in the food desire from NI to AI correlated negatively with changes in metabolism in pgACC/caudate in obese but not in non-obese men.

Conclusions Obese men showed higher activation in $\mathrm{pgACC} /$ caudate, which are regions involved with self-regulation and emotion/reward during AI. Behavioral associations suggest that successful AI is an active process requiring more energy in obese but not in non-obese men. The additional required effort to increase cognitive control in response to food stimulation in obese compared with non-obese men may contribute to their uncontrolled eating behavior.
\end{abstract}

Supplementary information The online version of this article (https:// doi.org/10.1038/s41366-019-0484-z) contains supplementary material, which is available to authorized users.

Gene-Jack Wang

gene-jack.wang@nih.gov

1 Laboratory of Neuroimaging, National Institute on Alcohol Abuse and Alcoholism, Bethesda, MD 20892-1013, USA

2 School of Life Science and Technology, Xidian University, Xi'an 710071 Shaanxi, China

3 Brookhaven National Laboratory, Upton, NY 11973, USA

4 National Institute on Drug Abuse, National Institutes of Health, Bethesda, MD 20892, USA

\section{Introduction}

Obesity is associated with significant increases in morbidity and mortality, highlighting the urgency for understanding the processes contributing to this epidemic [1,2]. In many of the current environments, highly palatable and affordable food is widely available. The capacity for self-regulation is likely to modulate an individual's risk for overeating. Indeed the capacity to regulate impulses, emotions, and desires has been linked to a broad spectrum of psychopathologies and problems including obesity [3-5].

We previously showed that the desire for food during presentation of palatable food stimuli was associated with striatal dopamine (DA) release, measured using positron emission tomography (PET) with $\left[{ }^{11} \mathrm{C}\right]$ raclopride, a radiotracer whose binding is sensitive to changes in 
extracellular DA. This finding is consistent with DA's role in modulating the motivation for food [6], which is mediated by its regulation of striato-cortical circuits involved with drive, salience attribution, and inhibitory control [7, 8]. Using the same experimental paradigm in normal-body-weight fasting subjects with 2-deoxy-2 $\left[{ }^{18} \mathrm{~F}\right]$ fluoro-D-glucose (FDG)-PET, we found that food presentation increased metabolism in orbitofrontal cortex (OFC) in proportion to the subjective perception of hunger and the desire to eat [9]. The OFC is implicated in controlling and planning behaviors and is regulated by DA both through direct as well as indirect striato-thalamo cortical projections. Indeed, a recent fMRI study using the blood oxygen level dependent signal showed that obese subjects activated striatum and OFC as well as insula (a brain region involved with interoception for food signals that is also innervated by DA terminals) while viewing pictures of high-caloric food [10].

Food is a potent natural reinforcer, the value of which is enhanced by food deprivation [11]. Understanding the neurobiological mechanisms underlying the inhibitory control of food intake when one desires it, may provide new targets for interventions to help individuals regulate their eating behaviors and maintain a healthy BMI. We previously reported on a PET study in normal weight participants in whom we measured regional brain glucose metabolic responses when exposed to appealing food both when directed to suppress the food desire (attempted inhibition or AI) and when exposed to the food but with no inhibition (NI) [12]. We showed that AI decreased the activation responses in anterior cingulate cortex (ACC), OFC, striatum and insula to food stimulation in men but not in women [12]. However, the implications of these findings to obesity remains elusive, particularly since healthy obese subjects have lower baseline (BL) metabolic activity in ACC and OFC that was associated with impaired performance on tests of cognitive control [13]. In this study, we used the same imaging paradigm as reported previously [12] in a group of obese $(n=16)$ and non-obese men $(n=$ 11). We hypothesized, first, obese individuals would have decreased activation in OFC, striatum, and insula but increases in ACC during AI, as compared with controls. Second, we expected differential metabolic responses to AI compared with NI based on group (i.e., a group-bycondition interaction in metabolic activity), based on several studies suggesting obesity-related differences in brain functional activity when regulating responses to food [10] (for a review, see [14]). Finally, in line with substantial individual differences in brain responses and outcomes during self-control for food $[15,16]$, we expected that metabolic activity changes in AI versus NI would be significantly associated with suppression of hunger and food desire in AI relative to NI.

\section{Materials and methods}

\section{Participants}

The Institutional Review Board at Stony Brook University/ Brookhaven National Laboratory approved the protocol. Written informed consents were obtained after the experimental procedure was explained and after the participants had read the consent form. Sixteen obese males (32 \pm 8.7 years old) with mean BMI of $38.6 \pm 7.2$ and 11 agematched non-obese males (BMI 24.2 \pm 2.5 ) were recruited for the study. Obese participants had a BMI of 30 or over: 12 of them with BMI range (31.1-38.9), four of them were considered morbidly obese (43.1-60). Whereas the BMI of non-obese was below 30, six non-obese participants were considered normal weight (20.2-24.5) and five non-obese participants were considered overweight (25.1-29.6). Participants with the following conditions were excluded: past or present history of eating disorders as per DSM IV, surgical/medical treatment for weight control, dependence on alcohol or other drugs of abuse (except for caffeine $<5$ cups/day or nicotine $<1 \mathrm{pack} / \mathrm{day}$ ), neurological or psychiatric disorder, use of prescription (psychiatric and/or non-psychiatric) medication(s) that can affect brain function in the past 2 weeks, medical conditions that may alter cerebral function, cardiovascular disease and diabetes, and head trauma with loss of consciousness of more than $30 \mathrm{~min}$. Urine screening tests for psychoactive drugs (including PCP, cocaine, amphetamine, opiates, barbiturates, benzodiazepine, and THC) were performed to corroborate absence of drug use. Sample size was justified based on a prior study with a similar number of participants in which there was sufficient statistical power for detecting food inhibition effects [12].

Prior to the imaging studies, participants were asked to fill out a questionnaire, which contained the following information: a rating of their interest in food; their favorite foods; food smells that stimulated their appetite; food smells that diminished their appetite; and a list of foods to rate for their preference on a scale from 1 to 10,10 being the highest. The food items included a variety of popular American meals, snacks and desserts (e.g., bacon-egg-cheese sandwich, cinnamon bun, pizza, hamburger with cheese, fried chicken, lasagna, barbecue ribs, ice cream, brownie, and chocolate cake). The ten food items with the highest ratings were presented to the participant on the imaging study day prior to the food stimulation to confirm their favorite choices and during the food stimulation condition. To assess the behavioral dimensions of eating, participants also completed the Three Factor Eating Questionnaire (TFEQ) [17], which has three main factors (disinhibition, cognitive restraint of eating, and susceptibility to food cues). Since the participants had to fast for 15 
or more hours before the PET imaging was done, these questionnaires were filled on a screening day under 'normal' non-stressful circumstances.

\section{Experimental paradigm}

Participants were scanned three times with FDG in three different days under the following conditions: (1) Day A: food stimulation started $15 \mathrm{~min}$ before FDG injection and continued for a total of $45 \mathrm{~min}$ while lying in the PET scanner. When the stimulation was completed, the participants were positioned for imaging and acquisition was started $35 \mathrm{~min}$ after FDG injection. The participants were instructed to observe and spontaneously react to the food stimuli (no inhibition: NI). (2) Day B: procedures were as for Day A except that participants were instructed to inhibit their food desire prior to the presentation of the food stimuli (attempted inhibition: AI). Prior to the study, participants were instructed how to practice their attempted inhibition (i.e., ignore, shift thoughts) to the food stimuli. (3) Day C: $\mathrm{BL}$ condition without food stimulation. The $\mathrm{A}, \mathrm{B}$, and $\mathrm{C}$ sequence was randomized so that for one third of the participants the first day was day A, for one third it was day B and for the other third it was day $\mathrm{C}$.

For day A and day B, the food was warmed to enhance the smell and the participants were presented with it so that they could view it and smell it by one of the investigators (M.J.). A cotton swab impregnated with the food was placed on their tongue so they could taste (but not swallow) it. A given food item was presented for $5 \mathrm{~min}$ and then it was exchanged for a new one. The tasting, smelling, and viewing of a given food item were continuous during the stimulation. The participants were asked to describe their favorite foods and how they like to eat them while they were presented with foods that they had reported as among their favorite ones. For all conditions, participants were asked to have their last meal at 7 P.M. the evening before the day of the study and were studied between 17 and $19 \mathrm{~h}$ after their last meal. We relied on the participants' selfreport to confirm that they had not eaten anything after their last meal as instructed. The participants arrived at the imaging center at about 8:30 A.M. on the day of the study and a nurse remained with them to ensure they refrained from food or caloric containing drinks prior to imaging, which started after 12 P.M.

\section{PET imaging}

Participants were scanned with FDG using a Siemens HR + PET scanner. Details on procedures for positioning of the participant, arterialized venous and venous catheterization, quantification of radiotracer and transmission and emission scans have been published [9]. Briefly, two intravenous lines were inserted and maintained with saline. Arterialized venous blood was obtained from a catheter placed in a dorsal hand vein. The hand was prewarmed to $48^{\circ} \mathrm{C}$ in a heating box, which ensured the shifting of arterial blood to the vein. The other catheter was in the antecubital region of the opposite arm for tracer injection. One emission scan (20 min) was taken 35 min after an intravenous injection of 4-6 mCi of FDG. During the study participants were positioned supine in the PET camera with their eyes open; the room was dimly lit and noise was kept to a minimum.

\section{Behavioral assessment}

During the PET studies participants were instructed to orally respond to each descriptor using a whole number between 1 and 10 for the self-report of hunger and food desire, which were obtained during food presentation in $5 \mathrm{~min}$ intervals for a total of $45 \mathrm{~min}$ (ten samples). The measures from 25 to $45 \mathrm{~min}$ where averaged (Supplementary Fig. 1).

\section{Data analysis}

PET images were reconstructed using filtered back projection (Hann filter with a $4.9 \mathrm{~mm}$ FWHM kernel). Cerebral glucose metabolic rate (CMRglu) images were computed using Sokoloff's model [18]. CMRglu images were transferred into MNI space in SPM8 (Wellcome Trust Centre for Neuroimaging, London) [19]. SPM8 was used to perform voxelwise analysis of variance (ANOVA) using the full factorial model to study the within-subject factor conditions (with BL, NI, and AI levels) and the between-subject factor of groups (obese and non-obese) and their interactions. An uncorrected threshold of $p=0.002$ and minimum cluster size of 200 was applied to the statistical maps in SPM8. When indicated, correction for multiple comparisons was performed using random field theory for cluster size relative to whole brain or a small volume.

Behavioral ratings of hunger and food desire were analyzed using a repeated measures ANOVA with condition (NI and $\mathrm{AI}$ ) as within-group factor, and group (obese and non-obese) as between-group factor, using SPSS version 25 (IBM). Pearson product moment correlation analyses were used to assess the association between the ratings of hunger and food desire and the condition-related changes in CMRglu.

\section{Results}

Table 1 provides demographics of the obese and non-obese group. Obese participants did not differ from the non-obese in age or years of education. The obese participants had 
higher weights and BMI, and scores higher on the TFEQ score (total), driven by their higher subscores in disinhibition and cognitive restraint of eating (see Table 1).

\section{Behavioral ratings}

The repeated measures ANOVA showed a significant effect of condition (NI and $\mathrm{AI}$ ) on rating of hunger ratings $\left(F_{1,25}=56.0, p<0.0001\right)$ and food desire $\left(F_{1,25}=50.0, p<\right.$ 0.0001). During AI, both non-obese and obese men had lower hunger $\left(t_{26}=7.3, p<0.0001\right)$ and food desire $\left(t_{26}=\right.$ $7.1, p<0.0001$ ) scores compared with NI (also see Supplementary Fig. 1). There was no effect of group and no interaction effect of group $\times$ condition on ratings of hunger or food desire (Table 2).

\section{Metabolic changes between baseline and non- inhibited food stimulation}

In the no-stimulation BL, CMRglu was lower in a cluster $(k=246)$ within the left medial and superior frontal gyri (BA 9, peak coordinates: $x=-24 \mathrm{~mm}, y=18 \mathrm{~mm}$, and $z=$ $36 \mathrm{~mm}$ ) in obese than non-obese men (Supplementary Fig. 2). Relative to BL, the food stimulation with no inhibition (NI), had increases in CMRglu in a range of cerebellar, cortical and subcortical regions (Table 3, Fig. 1a).

Table 1 Characteristics of study participants

\begin{tabular}{llll}
\hline & Obese & Non-obese & $p$ value \\
\hline Number of participants & $16 \mathrm{Men}$ & $11 \mathrm{Men}$ & \\
Age range (years old) & $21-46$ & $24-45$ & \\
Age mean (years old) & $31.9 \pm 8.7$ & $31.2 \pm 5.8$ & $\mathrm{NS}$ \\
Body weight range (lb) & $192-480$ & $137-200$ & \\
Body weight mean (lb) & $269 \pm 67$ & $166 \pm 20$ & $<0.001$ \\
BMI range $\left(\mathrm{kg} / \mathrm{m}^{2}\right)$ & $31.1-60$ & $20.2-29.6$ & \\
BMI mean $\left(\mathrm{kg} / \mathrm{m}^{2}\right)$ & $38.6 \pm 7.2$ & $24.4 \pm 2.6$ & $<0.001$ \\
Education (years) & $13.5 \pm 2.0$ & $14.2 \pm 2.2$ & $\mathrm{NS}$ \\
TFEQ total & $7.2 \pm 1.8$ & $5.6 \pm 1.8$ & 0.001 \\
TFEQ cognitive restraint of eating & $8.2 \pm 4.3$ & $4.4 \pm 3.3$ & 0.03 \\
TFEQ disinhibition & $8.4 \pm 3.5$ & $3.7 \pm 2.1$ & $<0.001$ \\
TFEQ hunger & $8.9 \pm 3.2$ & $6.7 \pm 3.5$ & 0.1 \\
\hline
\end{tabular}

These increases were significant in both obese and nonobese groups (Fig. 1b). However, there were no significant differences in food stimulation with NI induced metabolic increases between obese and non-obese groups.

\section{Metabolic changes between non-inhibited food stimulation and attempted inhibition}

Relative to NI, AI in both non-obese and obese groups decreased CMRglu in middle and superior temporal gyrus, right insula, right subgenual anterior cingulate (sgACC) and right medial orbitofrontal cortex (mOFC, Table 4, Fig. 2a), while the same regions had higher CMRglu in NI relative to BL (Fig. 2b).

\section{Interaction between brain glucose metabolism and attempted inhibition}

For the interaction between-group and experimental condition, we found a cluster (BA32) within the pgACC and caudate with differential involvement in NI versus AI for the obese and non-obese groups (Table 5, Fig. 3). In the non-obese group, we found significant increases from BL to $\mathrm{NI}$ and significant decreases from NI to AI in this cluster (Fig. 3b). In the obese group, however, we found significant decreases from BL to NI and increases from NI to AI in this cluster (Fig. 3b). There was no significant group interaction effect for CMRglu changes between BL and NI.

Interestingly, the CMRglu during AI in the left dorsolateral prefrontal cortex (DLPFC) cluster (with significant BL group differences), showed a trend correlation with the pgACC cluster across non-obese $(r(9)=0.59, p=0.056)$ but not across obese men $(r(14)=0.36, p=0.17)$.

\section{Correlation between brain glucose metabolism and behavioral ratings}

In the obese group, condition-related differences in ratings of hunger and food desire were associated with conditionrelated changes in CMRglu. Specifically, there was a negative association between changes in ratings from NI to $\mathrm{AI}$ and CMRglu changes in pgACC/caudate (Hunger: $R^{2}=$ $0.33, p=0.020$; Food desire: $R^{2}=0.35, p=0.015$; Fig. 4 ;
Table 2 Comparison between obese and non-obese men for the difference in the scores (from 1 to $10 ; 0$ is the least and 10 is the most) of self-report of hunger and food desire during food stimulation conditions

\begin{tabular}{|c|c|c|c|c|c|c|}
\hline & \multicolumn{2}{|l|}{ Hunger } & \multirow[b]{2}{*}{$p$ value } & \multicolumn{2}{|l|}{ Food desire } & \multirow[b]{2}{*}{$p$ value } \\
\hline & Obese & Non-OB & & Obese & Non-OB & \\
\hline No inhibition (NI) & $8.6 \pm 1.0$ & $8.8 \pm 0.8$ & NS & $8.3 \pm 1.1$ & $8.4 \pm 1.1$ & NS \\
\hline Attempted inhibition (AI) & $5.5 \pm 2.6$ & $4.5 \pm 2.9$ & NS & $5.1 \pm 2.5$ & $4.4 \pm 2.9$ & NS \\
\hline $\mathrm{AI}-\mathrm{NI}$ & $-3.2 \pm 2.3$ & $-4.3 \pm 2.9$ & NS & $-3.2 \pm 2.6$ & $-4.0 \pm 2.6$ & NS \\
\hline NI vs AI ( $p$ value) & $<0.001$ & $<0.001$ & & $<0.001$ & $<0.001$ & \\
\hline
\end{tabular}

NI food stimulation without attempted inhibition, $A I$ food stimulation with attempted inhibition 
Table 3 Food stimulation (without inhibition) versus baseline condition (both groups)

\begin{tabular}{|c|c|c|c|c|c|c|c|}
\hline Region(s) & $\mathrm{L} / \mathrm{R}$ & Brodmann area(s) & Cluster size & $\begin{array}{l}\text { Peak } \\
(x, y,\end{array}$ & $\begin{array}{l}\text { ordina } \\
\mathrm{mm}\end{array}$ & & $\begin{array}{l}\text { Peak } \\
t \text {-value }\end{array}$ \\
\hline $\begin{array}{l}\text { Cerebellum ant lobe } \\
\text { Cerebellum post lobe } \\
\text { Declive } \\
\text { Cuneus } \\
\text { Middle temporal gyrus } \\
\text { Sup. temporal gyrus } \\
\text { Calcarine } \\
\text { Tuber } \\
\text { Cerebellar tonsil } \\
\text { Precentral gyrus } \\
\text { Postcentral gyrus } \\
\text { Precuneus } \\
\text { Culmen }\end{array}$ & $\mathrm{L} / \mathrm{R}$ & 18,22 & $* 15345$ & -30 & -58 & -30 & 6.10 \\
\hline $\begin{array}{l}\text { Sup. temporal gyrus } \\
\text { Middle temporal gyrus } \\
\text { Inf. temporal gyrus }\end{array}$ & $\mathrm{L}$ & $21,38,20$ & $* 724$ & -38 & 14 & -32 & 4.18 \\
\hline $\begin{array}{l}\text { Middle temporal gyrus } \\
\text { Sup. temporal gyrus } \\
\text { Uncus } \\
\text { Inf. frontal gyrus } \\
\text { Parahippocampus }\end{array}$ & $\mathrm{R}$ & 38,47 & $* 365$ & 28 & 2 & -34 & 4.10 \\
\hline $\begin{array}{l}\text { Parahippocampus } \\
\text { Uncus }\end{array}$ & $\mathrm{L}$ & 34,28 & 206 & -18 & -14 & -24 & 4.01 \\
\hline $\begin{array}{l}\text { Inf. frontal gyrus } \\
\text { Sup. temporal gyrus }\end{array}$ & $\mathrm{R}$ & 47,38 & 261 & 54 & 24 & -2 & 4.05 \\
\hline $\begin{array}{l}\text { Sup. temporal gyrus } \\
\text { Inf. frontal gyrus } \\
\text { Middle temporal gyrus } \\
\text { Precentral gyrus } \\
\text { Postcentral gyrus } \\
\text { Insula } \\
\text { Middle frontal gyrus } \\
\text { Inf. parietal lobule }\end{array}$ & $\mathrm{L}$ & $22,40,21$ & *10042 & -56 & -12 & 24 & 7.01 \\
\hline $\begin{array}{l}\text { Thalamus } \\
\text { Medial dorsal nucleus } \\
\text { Midbrain }\end{array}$ & $\mathrm{L} / \mathrm{R}$ & - & $* 771$ & 6 & -18 & 4 & 4.35 \\
\hline Precuneus & $\mathrm{R}$ & 7,31 & 213 & 10 & -58 & 30 & 4.17 \\
\hline Superior frontal gyrus & $\mathrm{L} / \mathrm{R}$ & 6 & $* 391$ & -8 & 12 & 60 & 5.07 \\
\hline
\end{tabular}

$p=0.002$, uncorrected, minimum cluster size $=200$

$* p_{\text {FWE }}<0.05$ cluster-size corrected, random field theory also see Supplementary Fig. 3); such that greater suppression of appetite was associated with higher $\mathrm{pgACC} /$ caudate activation (cluster showing the interaction effect, Fig. 3; Table 5). In the non-obese participants, this association was not significant (Hunger: $R^{2}=0.002, p=0.896$; Food desire: $R^{2}=0.02, p=0.650$; Fig. 4).

In non-obese participants, for both hunger $\left(R^{2}=0.41\right.$, $p=0.034)$ and food desire $\left(R^{2}=0.38, p=0.045\right)$, lower ratings during $\mathrm{AI}$ were associated with lower $\mathrm{pgACC} / \mathrm{cau}-$ date metabolism (Fig. 5) for the cluster showing the interaction effect in Fig. 3. This is consistent with non-obese participants showing less activation in this cluster for AI than NI (Fig. 3, Table 5).
We did not observe a correlation between changes in CMRglu and the TFEQ-disinhibition and TFEQ-cognitive restraint of eating measures neither in the obese nor in the non-obese groups. However, the correlation between the TFEQ-hunger measures and CMRglu during AI in the regions activated during $\mathrm{AI}>\mathrm{NI}$ ( $\mathrm{sgACC}, \mathrm{mOFC}$, posterior insula/superior temporal gyrus, and middle temporal gyrus) revealed a negative association in obese but not in nonobese participants $\left(R^{2}=0.35, p=0.016\right.$; Fig. 6a). The TFEQ-hunger scores were also negatively associated with CMRglu in the cluster showing an interaction effect during $\mathrm{AI}$ (pgACC and caudate; $R^{2}=0.29, p=0.030$; Fig. $6 \mathrm{~b}$ ), in obese but not in non-obese participants. 

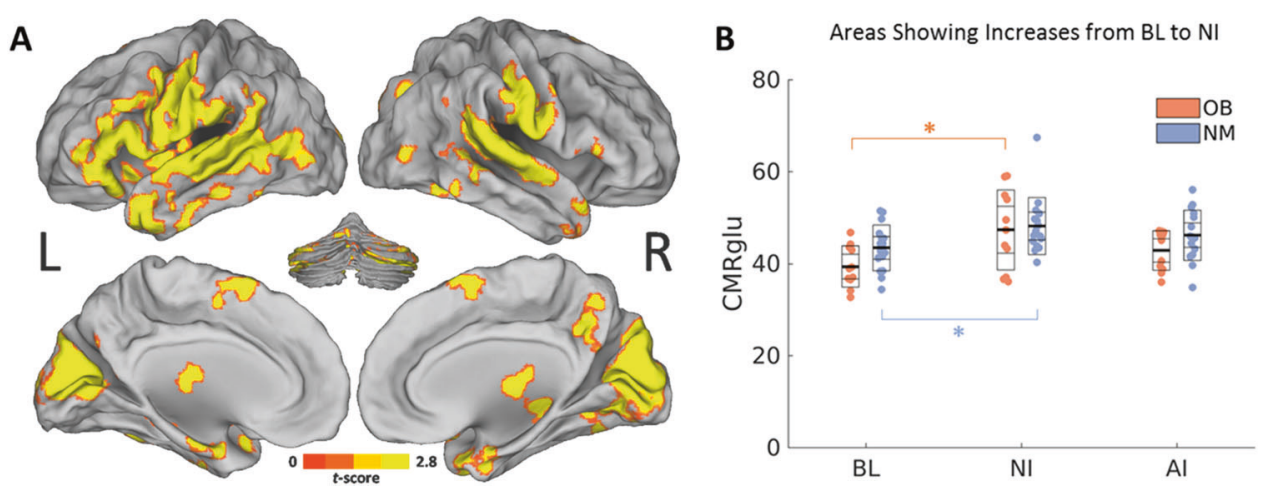

Fig. 1 Effect of food stimulation. a Brain regions showing CMRglu

see Table 1. b Group average of CMRglu within areas shown in a in increases in food stimulation with no inhibition (NI) relative to baseBL, no inhibition (NI), and attempted inhibition (AI) conditions. line $(\mathrm{BL}), p=0.002$ uncorrected, minimum cluster size $=200$. Also Significant differences are shown with brackets

Table 4 Attempted inhibition versus without inhibition to food stimulation condition (both groups)

\begin{tabular}{|c|c|c|c|c|c|c|c|}
\hline \multirow{2}{*}{$\begin{array}{l}\text { Region(s) } \\
\text { Middle temporal gyrus } \\
\text { Sup. temporal gyrus }\end{array}$} & \multirow{2}{*}{$\begin{array}{l}\mathrm{L} / \mathrm{R} \\
\mathrm{L}\end{array}$} & \multirow{2}{*}{$\begin{array}{l}\text { Brodmann area(s) } \\
22,21\end{array}$} & \multirow{2}{*}{$\begin{array}{l}\text { Cluster size } \\
* 381\end{array}$} & \multicolumn{2}{|c|}{$\begin{array}{l}\text { Peak } \\
\text { coordinates } \\
(x, y, z)_{\mathrm{mm}}\end{array}$} & \multicolumn{2}{|r|}{$\begin{array}{l}\text { Peak } \\
t \text {-value }\end{array}$} \\
\hline & & & & -48 & -38 & 2 & 4.58 \\
\hline $\begin{array}{l}\text { Medial frontal gyrus } \\
\text { Subgenual Ant. cingulate (sgACC) } \\
\text { Orbitofrontal gyrus }\end{array}$ & $\mathrm{R}$ & 32,10 & 215 & 20 & 34 & -4 & 4.02 \\
\hline $\begin{array}{l}\text { Sup. Temporal Gyrus } \\
\text { Insula }\end{array}$ & $\mathrm{R}$ & 41,13 & 215 & 56 & -38 & 18 & 4.11 \\
\hline
\end{tabular}

$p=0.002$, uncorrected, minimum cluster size $=200$

$* p_{\text {FWE }}<0.05$ cluster-size corrected, random field theory
Fig. 2 Effect of attempted inhibition (AI) in non-obese (NM) and obese (OB) individuals. a Brain regions showing decreases in CMRglu in active inhibition (AI) relative to no inhibition (NI) in both groups $(p=0.002$ uncorrected, minimum cluster size $=200$ ). Also see Table 2. b Group average of CMRglu within areas shown in a in BL, no inhibition (NI), and attempted inhibition (AI) conditions
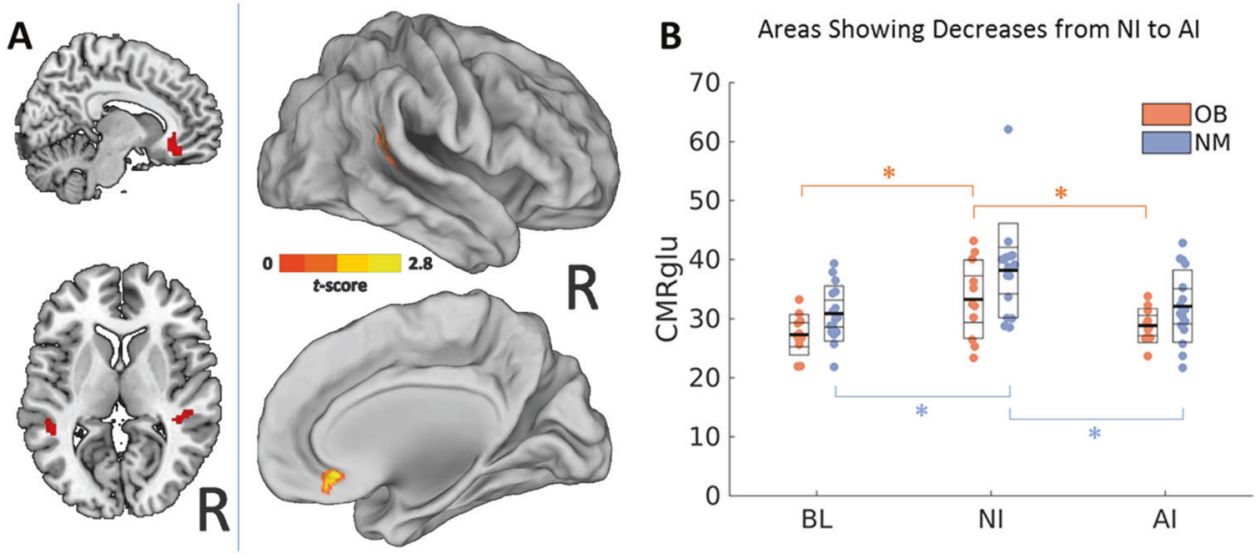

\section{Discussion}

This study explored the regional brain metabolic activity, which is presumed to reflect brain activity during food stimulation with and without attempted inhibition in obese men compared their response with those in non-obese men. The findings show that while brain responses to food stimulation with NI were similar between obese and nonobese men, regional brain responses to AI of food craving differed between the obese and the non-obese group. We discuss these findings in more detail below.

\section{Effects of food stimulation without inhibiting food craving}

The SPM comparison between BL and food stimulation (NI) conditions showed that both groups significantly increased CMRglu in what we describe as a "food 
stimulation network" that included inferior and middle frontal and temporal cortices, precuneus, insula, thalamus, and cerebellum. This finding replicates our previous work [9], and suggests that there may not be significant differences between non-obese and obese men in the regional brain's metabolic responses to the combination of viewing, smelling and tasting food generally. Participants in both groups fasted for 15 or more hours before the PET FDG scan, which may have minimized group differences in hunger drive. Thus, the positive finding in the current study is supportive of an actual effect of obesity on brain glucose metabolism responses.

\section{Effects of food stimulation during attempted inhibition of food craving}

Similar to our prior findings in non-obese males [12], obese participants showed lower CMRglu in sgACC/mOFC (BA

Table 5 Interaction between attempted inhibition and without inhibition to food stimulation between two groups

\begin{tabular}{|c|c|c|c|c|c|}
\hline Region(s) & $\mathrm{L} / \mathrm{R}$ & $\begin{array}{l}\text { Brodmann } \\
\text { area }(\mathrm{s})\end{array}$ & Cluster size & $\begin{array}{l}\text { Peak } \\
\text { coordi- } \\
\text { nates } \\
(x, y, z) \\
\mathrm{mm}\end{array}$ & $\begin{array}{l}\text { Peak } \\
t \text {-value }\end{array}$ \\
\hline $\begin{array}{l}\text { Pregenual } \\
\text { Ant. Cingulate } \\
\text { (pgACC) } \\
\text { Caudate }\end{array}$ & $\mathrm{R}$ & 32 & ${ }^{a} 278$ & $20 \quad 38$ & 5.18 \\
\hline
\end{tabular}

$p=0.002$, uncorrected, minimum cluster size $=200$

${ }^{a}$ Correction for multiple comparisons using small volume correction in a sphere with a $50 \mathrm{~mm}$ radius within the frontal cortex, $p_{\mathrm{FWE}}<0.05$

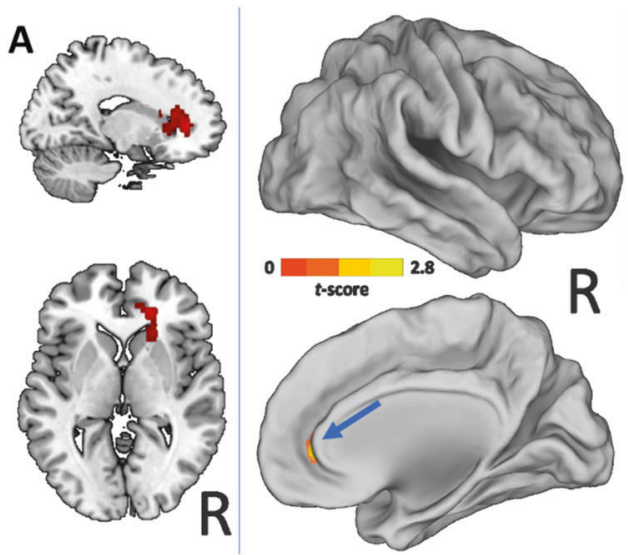

Fig. 3 Interaction between inhibition to food stimulation and group factors in CMRglu. a Brain regions showing different changes from food stimulation with no inhibition (NI) to food stimulation with attempted inhibition (AI) in normal controls (NM) versus obese (OB)
$32,10)$, and bilateral posterior insula/superior temporal and middle temporal gyri during AI compared with NI. These regions are notable for their involvement in multisensory integration, as well as reward and emotional processing [20-22]. The sgACC is of particular interest, since it serves as a functional nexus for reward, emotion, and cognitive processes [23]. For instance, when a cognitive control task was carried out in an emotional context or when the emotional stimuli were relevant to the cognitive task being carried out, the sgACC was activated [24]. The ventral medial PFC is also involved in self-control and decision making processes as part of a circuit including DLPFC. The sgACC encodes stimulus values that guide decisions at the time of choice, relaying these values to dLPFC [25]. The DLPFC could filter sensory input then modulate and incorporate these value signals for successful cognitive control during decision making, making this a likely circuit that regulates food intake behavior.

Empirical studies have shown support for this possibility. For example, decision task studies suggest that the DLPFC influences self-control by modulating $\operatorname{sgACC}$ value signals to reflect more desirable elements such as the health value, rather than more attractive but inferior rewards such as taste value of foods [26-28]. Based on this work, our observed suppression of sgACC CMRglu during AI in the non-obese group may reflect suppressed hunger and food desire. Notably, in this study, the food stimulation was not passive viewing of food cues but actively viewing, smelling and tasting preferred food items simultaneously that were individually selected by each participant prior to study day. This multisensory stimulation procedure recruited brain regions involved with sensory processing, emotional regulation, conditioning and motivation, and these same regions were suppressed during AI.
B Areas Showing Group-by-Condition Interaction

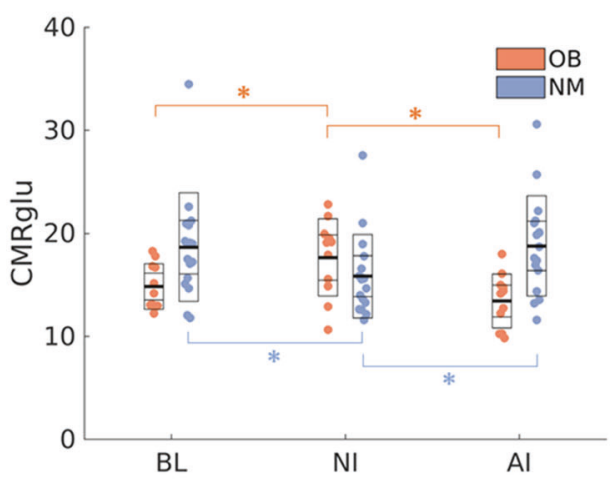

participants ( $p=0.002$ uncorrected, minimum cluster size $=200$ ). Also see Table 3. b Group average of CMRglu within areas shown in a in BL, no inhibition (NI), and attempted inhibition (AI) conditions 


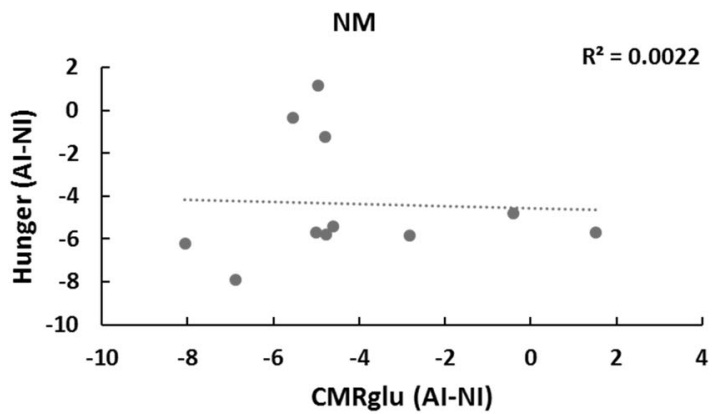

$\mathrm{OB}$

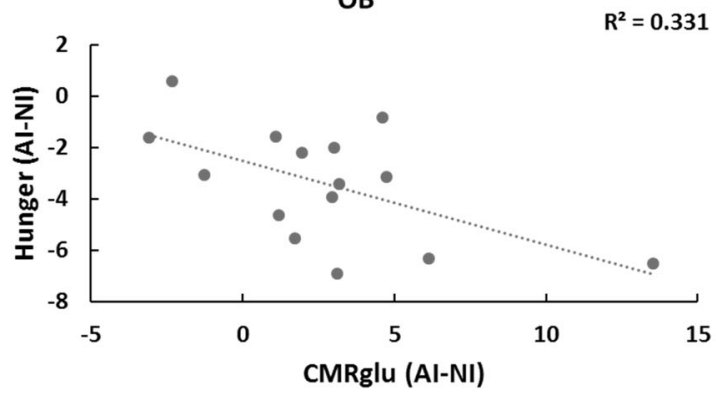

Fig. 4 Correlation between changes in the behavioral measures and CMRglu in the cluster showing interaction effect between-group and inhibition to food stimulation factors (Fig. 3). One outlier participant for behavioral assessment was excluded (see Supplementary Fig. 2).
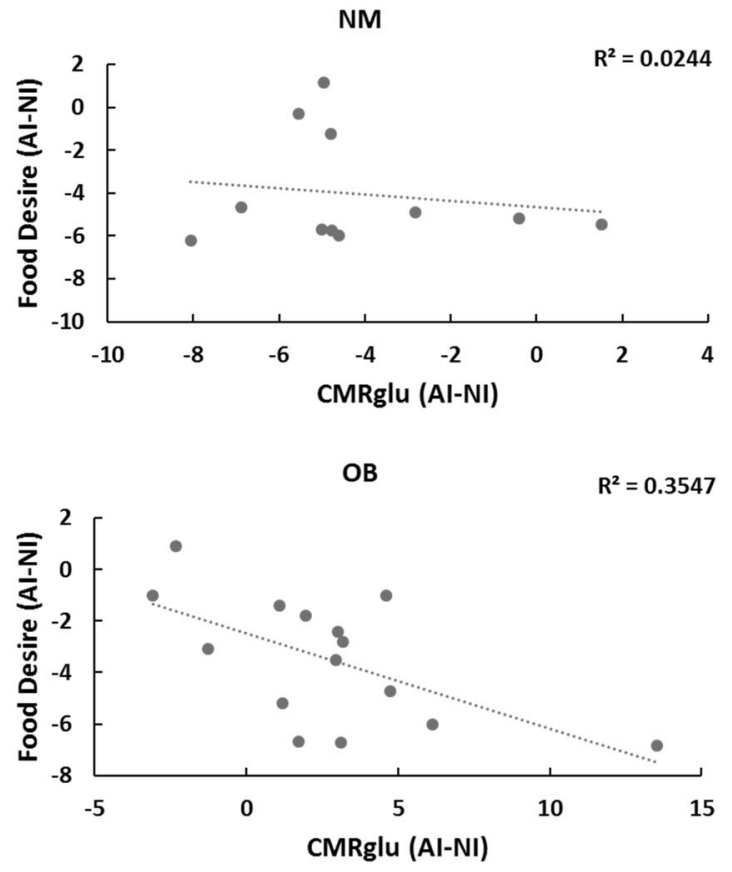

NI food stimulation without inhibition, AI food stimulation with attempted inhibition, OB obese, and NM non-obese controls. Both correlations were significant for the $\mathrm{OB}$ group. OB participants with more hunger suppression increased CMRglu
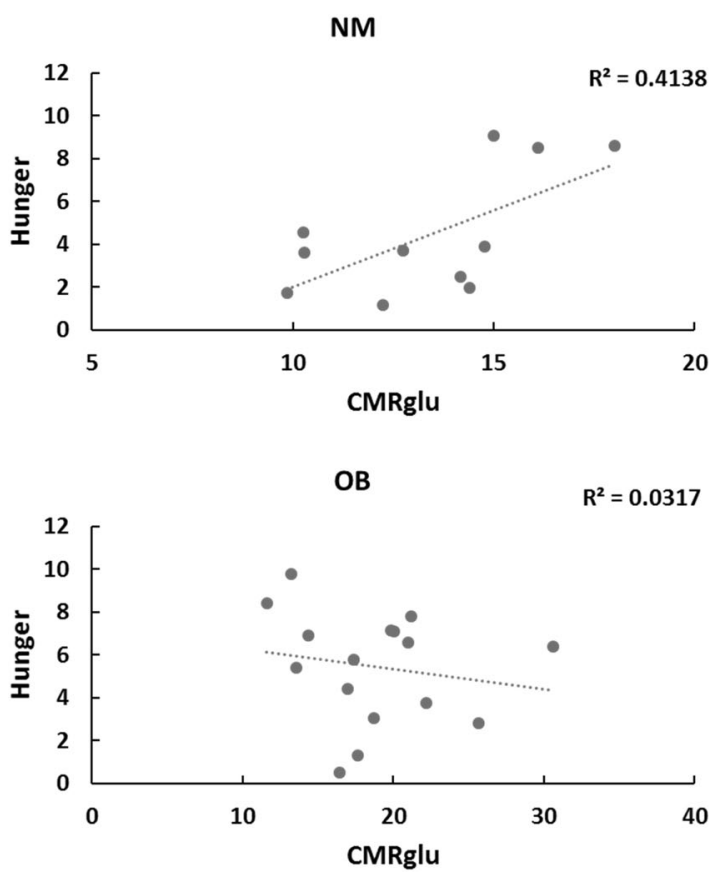

Fig. 5 Correlations between behavioral measures and CMRglu during $\mathrm{AI}$ in the cluster showing interaction effect between-group and inhibition to food stimulation factors (Fig. 3). NI food stimulation without

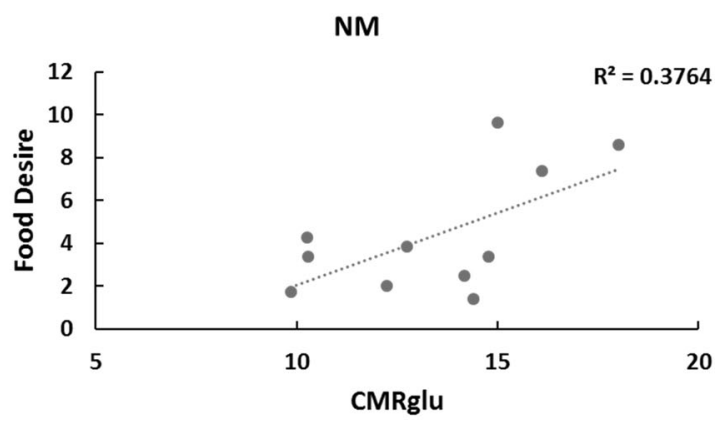

OB

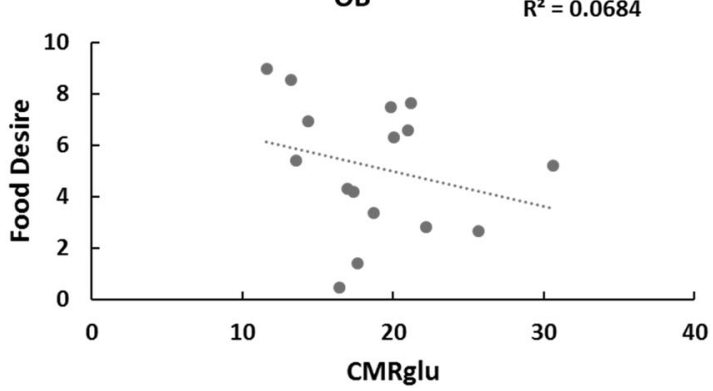

inhibition, AI food stimulation with attempted inhibition, OB obese, and NM non-obese controls. Both correlations were significant only for the NM group 


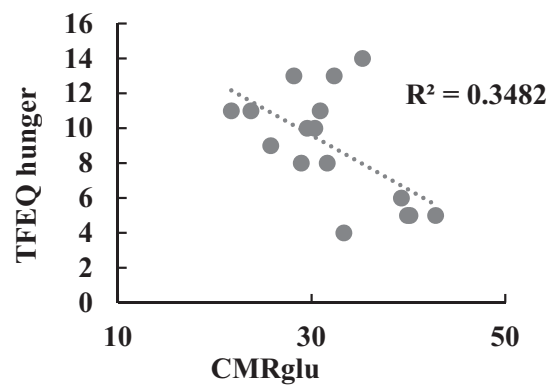

Fig. 6 Association between TFEQ-hunger measures and CMRglu changes in food inhibition network (including sgACC, OFC, insula, and temporal cortex) during $\mathrm{AI}$ condition (Fig. 6a) and with CMRglu

\section{Interactive effects of obesity and inhibition of food craving on brain glucose metabolism}

For the interaction between-group and inhibition conditions, we found a cluster including parts of the pgACC and caudate that had differential involvement in NI versus AI for obese and non-obese groups. Specifically, in nonobese participants, this cluster was significantly increased from BL to NI and decreased from NI to AI, whereas OB participants increased CMRglu in this region from NI to AI. The pgACC area is known to be activated by many reward related stimuli including fat texture and sweet taste [22, 29]. This region involves action outcome learning process and is activated when an expected reward delays [30]. The caudate is associated with reward anticipation [31, 32] and initiation of eating behavior [33], which has been shown to be activated in patients with binge eating disorder during food stimulation condition using the same food stimulation paradigm of this study [34]. The increases of CMRglu in pgACC/caudate cluster of obese participants could reflect greater energy need in this region during the inhibition control task. In obese men (but not non-obese), changes in the rating of hungry and food desire ratings from NI to AI was negatively correlated with CMRglu changes in the pgACC/ caudate cluster showing an interaction effect. Thus, successful AI in obese participants was related to recruitment of additional metabolic activation in this cluster. Decreased CMRglu in frontal cortex has been shown in obese participants, which is associated with decreased cognitive function [13]. The decreases of CMRglu in PFC (including ACC) in morbidly obese participants was associated with deficits in DA D2 receptor mediated striatal signaling (including caudate) [8]. In this study, we also observed that the obese participants had lower BL CMRglu in left DLPFC. We speculate that obese participants have greater difficulty in suppressing their desire to eat during food stimulation, for it is a more demanding cognitive effort that appears to require a greater energetic

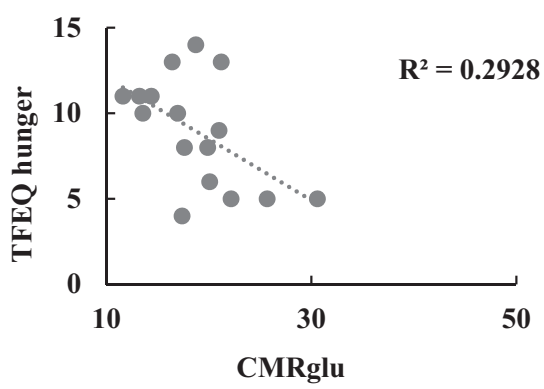

changes for interaction effect (including pgACC and caudate) between groups and inhibition condition (Fig. 6b) in obese participants

cost for activating the $\mathrm{pgACC} /$ caudate region which is needed for self-regulation [35].

\section{Behavioral associations with CMRglu changes during food stimulation and inhibition}

In our study, the obese group had higher TFEQdisinhibition and TFEQ-restraint scores than the nonobese group, but these measures did not correlate with CMRglu. On the other hand, though TFEQ-hunger scores did not differ between the groups, in obese but not in non-obese participants, they were negatively associated with CMRglu during AI in the "food inhibition network" (regions that differed between $\mathrm{AI}$ and NI). Activation of sgACC was reported in an fMRI study that compared participants with different TFEQ-disinhibition scores when assessing valuation (greater taste-unhealthy vs. neutral taste-healthy) of food [36]. In this study, participants with lower disinhibition scores attenuated sgACC activity when fed whereas participants with higher disinhibition scores showed enhanced activity. In our study obese men had higher TFEQ-disinhibition scores, which is consistent with prior reports and expected for these scales, which were designed to measure long-term attitudes to eating [37]. The measures of TFEQ-hunger have been associated with a measure of susceptibility to hunger cues. It is likely that obese participants are more susceptible to craving when exposed to food cues under fasting condition, and this craving may be reflected in the regional patterns of brain glucose metabolism observed here.

\section{Limitations}

Because our prior study demonstrated that female participants did not suppress CMRglu during AI using the same food stimulation paradigm [12], we recruited male participants only in the present study. Thus, the findings in this study may not generalize to female obese participants. 
Future studies using different food stimulation paradigms are warranted to understand gender differences on attempted inhibition and its effect on food cue induced brain activation. Moreover, we investigated presession food intake using self-reports on the TFEQ. However, a newer version is available that has better validity and facture structure than the TFEQ used in the current study [38]. Furthermore, study data collection took part between 2011 and 2012. As a result, principles and practices of open science including preregistration of hypotheses and planned analyses were not adhered to. In addition, Future studies should attempt to replicate our findings in a larger and more diverse sample of participants. Last, the non-obese group consisted of participants in both the normal weight (BMI 20-25) and overweight (25-30) category.

\section{Summary}

Here we show the suppression of CMRglu in sgACC/OFC, posterior insula and superior and middle temporal gyri during food presentation with AI of food craving in non-obese men. These regions are involved in sensory processing, emotional regulation, conditioning, and motivation, suggesting their role in the neurobiological mechanisms underlying cognitive inhibition of the desire for food. Interestingly, the pgACC/ caudate regions in obese men showed an increase in CMRglu from NI to AI (a change that was correlated with better suppression scores), while the non-obese men showed an opposite pattern. The pgACC/caudate regions are involved in processing motivation for food consumption. The additional required effort to increase cognitive control in response to food stimulation in obese compared with non-obese men may contribute to their uncontrolled eating behavior.

Acknowledgements The PET study was carried out at Brookhaven National Laboratory with a support in part from Intramural Research Program of the National Institute on Alcoholism and Alcohol Abuse (Y01AA3009). We thank Karen Apelskog-Torres for study protocol preparation, David Schlyer and Michael Schueller for cyclotron operations; Donald Warner, David Alexoff and Paul Vaska for PET operations; Colleen Shea, Youwen Xu, Lisa Muench and Payton King for radiotracer preparation and analysis, Millard Jayne (MJ) for participant recruitment and performing food stimulation, Frank Telang, Barbara Hubbard and Pauline Carter for patient care.

\section{Compliance with ethical standards}

Conflict of interest The authors declare that they have no conflict of interest.

Publisher's note Springer Nature remains neutral with regard to jurisdictional claims in published maps and institutional affiliations.

Open Access This article is licensed under a Creative Commons Attribution 4.0 International License, which permits use, sharing, adaptation, distribution and reproduction in any medium or format, as long as you give appropriate credit to the original author(s) and the source, provide a link to the Creative Commons license, and indicate if changes were made. The images or other third party material in this article are included in the article's Creative Commons license, unless indicated otherwise in a credit line to the material. If material is not included in the article's Creative Commons license and your intended use is not permitted by statutory regulation or exceeds the permitted use, you will need to obtain permission directly from the copyright holder. To view a copy of this license, visit http://creativecommons. org/licenses/by/4.0/.

\section{References}

1. Ghanta RK, LaPar DJ, Zhang Q, Devarkonda V, Isbell JM, Yarboro LT, et al. Obesity increases risk-adjusted morbidity, mortality, and cost following cardiac surgery. J Am Heart Assoc. 2017;6:e003831.

2. Yatsuya H, Li Y, Hilawe EH, Ota A, Wang C, Chiang C, et al. Global trend in overweight and obesity and its association with cardiovascular disease incidence. Circ J. 2014;78:2807-18.

3. Volkow ND, Wang GJ, Tomasi D, Baler RD. The addictive dimensionality of obesity. Biol Psychiatry. 2013;73:811-8.

4. Volkow ND, Wang GJ, Tomasi D, Baler RD. Obesity and addiction: neurobiological overlaps. Obes Rev. 2013;14:2-18.

5. Lykouras L. Psychological profile of obese patients. Dig Dis. 2008;26:36-9.

6. Martel P, Fantino M. Mesolimbic dopaminergic system activity as a function of food reward: a microdialysis study. Pharmacol Biochem Behav. 1996;53:221-6.

7. Bassareo V, De Luca MA, Di Chiara G. Differential expression of motivational stimulus properties by dopamine in nucleus accumbens shell versus core and prefrontal cortex. J Neurosci. 2002;22:4709-19.

8. Volkow ND, Wang GJ, Telang F, Fowler JS, Thanos PK, Logan J, et al. Low dopamine striatal D2 receptors are associated with prefrontal metabolism in obese subjects: possible contributing factors. Neuroimage. 2008;42:1537-43.

9. Wang GJ, Volkow ND, Telang F, Jayne M, Ma J, Rao M, et al. Exposure to appetitive food stimuli markedly activates the human brain. Neuroimage. 2004;21:1790-7.

10. Rothemund Y, Preuschhof C, Bohner G, Bauknecht HC, Klingebiel R, Flor H, et al. Differential activation of the dorsal striatum by high-calorie visual food stimuli in obese individuals. Neuroimage. 2007;37:410-21.

11. Yousuf M, Heldmann M, Gottlich M, Munte TF, Donamayor N. Neural processing of food and monetary rewards is modulated by metabolic state. Brain Imaging Behav. 2018;12:1379-92.

12. Wang GJ, Volkow ND, Telang F, Jayne M, Ma Y, Pradhan K, et al. Evidence of gender differences in the ability to inhibit brain activation elicited by food stimulation. Proc Natl Acad Sci USA. 2009;106:1249-54.

13. Volkow ND, Wang GJ, Telang F, Fowler JS, Goldstein RZ, AliaKlein $\mathrm{N}$, et al. Inverse association between BMI and prefrontal metabolic activity in healthy adults. Obesity. 2009;17:60-5.

14. Kumar S, Grundeis F, Brand C, Hwang HJ, Mehnert J, Pleger B. Differences in insula and pre-/frontal responses during reappraisal of food in lean and obese humans. Front Hum Neurosci. 2016;10:233.

15. Hays NP, Roberts SB. Aspects of eating behaviors "disinhibition" and "restraint" are related to weight gain and BMI in women. Obesity. 2008;16:52-8.

16. Lopez RB, Courtney AL, Wagner DD. Recruitment of cognitive control regions during effortful self-control is associated with altered brain activity in control and reward systems in dieters during subsequent exposure to food commercials. Peer J. 2019;7:e6550. 
17. Stunkard AJ, Messick S. The three-factor eating questionnaire to measure dietary restraint, disinhibition and hunger. J Psychosom Res. 1985;29:71-83.

18. Sokoloff L, Reivich M, Kennedy C, Des Rosiers M, Patlak CS, Pettigrew K, et al. The [14C] deoxyglucose method for the measurement of local cerebral glucose utilization: theory, procedure, and normal values in the conscious and anesthetized albino rat. J Neurochem. 1977;28:897-916.

19. Friston KJ, Holmes AP, Poline JB, Grasby PJ, Williams SC, Frackowiak RS, et al. Analysis of fMRI time-series revisited. NeuroImage. 1995;2:45-53.

20. Nomi JS, Schettini E, Broce I, Dick AS, Uddin LQ. Structural connections of functionally defined human insular subdivisions. Cereb Cotex. 2018;28:3445-56.

21. Peelle JE, Sommers MS. Prediction and constraint in audiovisual speech perception. Cortex. 2015;68:169-81.

22. Rolls ET. Reward Systems in the Brain and Nutrition. Annu Rev Nutr. 2016;36:435-70.

23. Azab H, Hayden BY. Correlates of economic decisions in the dorsal and subgenual anterior cingulate cortices. Eur J Neurosci. 2018;47:979-93.

24. Palomero-Gallagher N, Eickhoff SB, Hoffstaedter F, Schleicher A, Mohlberg H, Vogt BA, et al. Functional organization of human subgenual cortical areas: Relationship between architectonical segregation and connectional heterogeneity. Neuroimage. 2015;115:177-90.

25. Hare TA, Camerer CF, Rangel A. Self-control in decision-making involves modulation of the vmPFC valuation system. Science. 2009;324:646-8.

26. Harris A, Hare T, Rangel A. Temporally dissociable mechanisms of self-control: early attentional filtering versus late value modulation. J Neurosci. 2013;33:18917-31.

27. Hutcherson CA, Plassmann H, Gross JJ, Rangel A. Cognitive regulation during decision making shifts behavioral control between ventromedial and dorsolateral prefrontal value systems. J Neurosci. 2012;32:13543-54.

28. van Steenbergen H, Watson P, Wiers RW, Hommel B, de Wit S. Dissociable corticostriatal circuits underlie goal-directed vs. cue- elicited habitual food seeking after satiation: evidence from a multimodal MRI study. Eur J Neurosci. 2017;46:1815-27.

29. Grabenhorst F, Rolls ET, Bilderbeck A. How cognition modulates affective responses to taste and flavor: top-down influences on the orbitofrontal and pregenual cingulate cortices. Cereb Cortex. 2008;18:1549-59.

30. Kable JW, Glimcher PW. The neural correlates of subjective value during intertemporal choice. Nat Neurosci. 2007;10:1625-33.

31. Salimpoor VN, Benovoy M, Larcher K, Dagher A, Zatorre RJ. Anatomically distinct dopamine release during anticipation and experience of peak emotion to music. Nat Neurosci. 2011;14:257-62.

32. Benningfield MM, Blackford JU, Ellsworth ME, Samanez-Larkin GR, Martin PR, Cowan RL, et al. Caudate responses to reward anticipation associated with delay discounting behavior in healthy youth. Dev Cogn Neurosci. 2014;7:43-52.

33. Szczypka MS, Kwok K, Brot MD, Marck BT, Matsumoto AM, Donahue BA, et al. Dopamine production in the caudate putamen restores feeding in dopamine-deficient mice. Neuron. 2001;30:819-28.

34. Wang GJ, Geliebter A, Volkow ND, Telang FW, Logan J, Jayne $\mathrm{MC}$, et al. Enhanced striatal dopamine release during food stimulation in binge eating disorder. Obesity. 2011;19:1601-8.

35. Jones CL, Minati L, Nagai Y, Medford N, Harrison NA, Gray M, et al. Neuroanatomical substrates for the volitional regulation of heart rate. Front Psychol. 2015;6:300.

36. Lee Y, Chong MF, Liu JC, Libedinsky C, Gooley JJ, Chen S, et al. Dietary disinhibition modulates neural valuation of food in the fed and fasted states. Am J Clin Nutr. 2013;97:919-25.

37. Blundell JE, Stubbs RJ, Golding C, Croden F, Alam R, Whybrow $\mathrm{S}$, et al. Resistance and susceptibility to weight gain: individual variability in response to a high-fat diet. Physiol Behav. 2005;86:614-22.

38. Karlsson J, Persson LO, Sjostrom L, Sullivan M. Psychometric properties and factor structure of the Three-Factor Eating Questionnaire (TFEQ) in obese men and women. Results from the Swedish Obese Subjects (SOS) study. Int J Obes Relat Metabol Disord. 2000;24:1715-25. 